\title{
MOTIVACIÓN EN EL TENIS: UN ESTUDIO CON JÓVENES TENISTAS
}

\author{
Angela Lope Alvarez \\ José Luis Antoñanzas Laborda \\ Universidad de Zaragoza \\ https://doi.org/10.17060/ijodaep.2017.n1.v3.1011
}

Fecha de Recepción: 14 Marzo 2017

Fecha de Admisión: 1 Abril 2017

\section{RESUMEN}

Uno de los principales factores que influyen en la actividad deportiva, tanto para su realización o ejecución como su continuidad es el llamado factor motivacional. Los distintos objetivos y metas que tienen los jóvenes tenistas, son analizados en este estudio con deportistas que abracan las edades y categorías desde benjamín hasta cadetes y junior. El saber jugar bien, mejorar mucho en esta disciplina junto con divertirse forman parte de los objetivos y metas de los tenistas desde edades tempranas.

Palabras clave: tenis, factores psicológicos, motivación, necesidades, entrenador

\section{INTRODUCCIÓN}

El tenis es conocido por su carácter elitista pero más todavía es conocido por la fortaleza mental que tienen que tener sus jugadores ya que la mayor parte del tiempo se encuentran solos en el terreno de juego (Romero, 2005).

Por tanto, aspectos como la motivación resultan de crucial importancia en tenistas tanto principiantes como sobre todo en profesionales ya que estas marcan la implicación que tiene el tenista tanto en los entrenamientos como en los partidos (Cervelló, Santos-Rosa, Jiménez, Nerea y García, 2002).

En cuanto a las motivaciones en el inicio de la práctica deportiva encontramos que en la investigación de González, Tabernero y Márquez (2000) mediante la utilización del "Participation Motivation Inventory" (Gill y cols., 1983) con una muestra de participantes que practican tanto futbol como tenis, en estos últimos, encontraron determinante la influencia de factores como la diversión o aspectos de la ejecución como mejorar sus habilidades mientras que cuando el nivel de competición aumenta los tenistas valoran más las recompensas extrínsecas como sentirse importante, alcanzar un status o ganar (González, Tabernero y Márquez, 2000).

También pueden encontrarse diversas orientaciones que se ponen de manifiesto en situaciones deportivas y de logro, estas son la orientación a la tarea y la orientación al logro (Nicholls, 1989). 


\section{MOTIVACIÓN EN EL TENIS: UN ESTUDIO CON JÓVENES TENISTAS}

En función de cuál de ellas este primando su competencia deportiva será diferente. Cuando el deportista tiene una alta orientación a la tarea se sentirá exitoso cuando consigue el dominio de la tarea que está realizando mientras que una alta orientación al ego su éxito será juzgado en función de su superioridad con aquellos sujetos con los que está compitiendo o comparándose. La orientación a la tarea se ha relacionado positivamente con la motivación intrínseca mientras que la orientación al ego se ha relacionado con la regulación introyectada y la regulación externa (Balaguer, Castillo, Duda y García-Merita, 2011).

Otra teoría a tener en cuenta es la Teoría de la Autodeterminación (Deci y Ryan, 1985), que constituye un modelo explicativo de la motivación humana con diversas aplicaciones de la que nos interesa especialmente la aplicación a la actividad física y deportiva. Sus aplicaciones han ido sobre todo encaminadas a una mejora en la motivación intrínseca (Moreno y Martínez, 2006).

\section{MÉTODO}

\section{Muestra}

Participaron en nuestro estudio un total de 61 sujetos (29 mujeres y 32 hombres). Nuestra muestra estaba compuesta por tenistas de competición de diferentes categorías del club, de benjamín a cadete, quedando representados de la siguiente manera: Benjamín (9-10 años) 8,2\%, Alevín (11-12 años) 34\%, Infantil (13-14 años) 27,9\%, Cadete (15-16 años) 21,3\% y Juvenil (18-19 años) $8,2 \%$.

Todos ellos fueron informados previamente mediante un consentimiento informado en el que se les comunicaba a los padres de los tenistas el propósito de la investigación y se solicitaba su colaboración, que tenían que devolver firmado.

\section{Procedimiento}

Los participantes fueron evaluados a través de un cuestionario creado ad hoc expresamente para la presente investigación. Dicho cuestionario contenía preguntas dirigidas a conocer aspectos relacionados con el tenis, la motivación y otros aspectos relacionados con la práctica de este deporte y las aportaciones que este deporte tiene en los tenistas. En cada una de las preguntas tenían que responder cual era la afirmación que más se ajustara a su forma de pensar

Los sujetos realizaron el cuestionario antes de la sesión de entrenamiento para controlar el cansancio y la distracción de los tenistas en una sala habilitada en el club a tales efectos.

Cuando todos los participantes de cada categoría habían llegado se les sentaba en la sala y se les entregaba el cuestionario, el investigador explicaba las instrucciones del mismo de forma grupal y permanecía en la sala contigua por si a alguno de los participantes le surgía cualquier duda poder resolvérsela en el momento.

\section{Resultados}

Los datos de los 61 sujetos fueron codificados y analizados mediante el paquete estadístico SPSS 15.0. Se analizaron las frecuencias de todas las variables del cuestionario ya que al tratarse de un estudio de carácter exploratorio se buscaba conocer cuáles eran las características de la muestra utilizada para posteriormente analizar las relaciones entre variables mediante correlaciones de Pearson.

En primer lugar se analizaron las frecuencias de las respuestas obtenidas a la pregunta ¿Cuál es la razón principal por la que empezaste a jugar a tenis? Se obtuvieron las siguientes frecuencias: para pasármelo bien y porque me gusta el tenis $(80,3 \%)$, realizar deporte $(9,8 \%)$ y porque jugaban mis amigos, familia... $(9,9 \%)$. 
En segundo lugar, los resultados de las correlaciones de Pearson. La tabla 3 presenta las correlaciones entre motivación:

Motivación 1: "ganar más partidos"

Motivación 2: "mejorar mucho"

Motivación 3: "entrenar con niños mejores"

Motivación 4: "competir"

Motivación 5: "ser el mejor"

Y los siguientes objetivos:

Objetivo 1: "me divierto"

Objetivo 2: "me resulta gratificante"

Objetivo 3: "para saber jugar mejor"

Objetivo 4: "para llegar a ser tenista"

Objetivo 5: "porque me encanta ir a entrenar"

Objetivo 6: "porque me gusta entrenar y competir"

Tabla 1.

Correlación Pearson entre motivación y objetivos

\begin{tabular}{|c|c|c|c|c|c|c|c|}
\hline & & objetivos 1 & objetivos 2 & objetivos 3 & objetivos 4 & objetivos 5 & objetivos 6 \\
\hline \multirow[t]{2}{*}{ motivacion 1} & Correlación de Pearson &,- 155 &,- 116 &, 105 &, 200 &,- 124 &,- 004 \\
\hline & Sig. (bilateral) &, 232 &, 373 &, 421 &, 123 & ,340 & ,976 \\
\hline \multirow[t]{2}{*}{ motivacion 2} & Correlación de Pearson &, 190 &,- 019 &, $313(*)$ &,- 074 &, 117 & ,199 \\
\hline & Sig. (bilateral) &, 143 &, 885 &, 014 & ,569 &, 369 & ,124 \\
\hline \multirow[t]{3}{*}{ motivacion 3} & Correlación de Pearson &, 162 &, $263(*)$ & ,208 &, $263(*)$ &, 219 &, $324(*)$ \\
\hline & Sig. (bilateral) &, 213 &, 041 &, 108 &, 040 &, 090 &, 011 \\
\hline & $\mathrm{N}$ & 61 & 61 & 61 & 61 & 61 & 61 \\
\hline motivacion 4 & Correlación de Pearson &, 162 &, $263(*)$ &, $279(*)$ &, 186 &, 219 &, 252 \\
\hline \multirow{2}{*}{ motivacion 5} & Sig. (bilateral) &, 210 & ,246 &, 758 &, 000 &, 850 &, 516 \\
\hline & $\mathrm{N}$ & 61 & 61 & 61 & 61 & 61 & 61 \\
\hline
\end{tabular}

* La correlación es significante al nivel 0,05 (bilateral).

** La correlación es significativa al nivel 0,01 (bilateral).

De las correlaciones que han resultado estadísticamente significativas, destacamos la correlación entre "ser el mejor" (motivación 5) con "para llegar a ser tenista" (objetivos 4) con una correlación media de 0,494 y una significación de 0.01 .

En cuanto a las correlaciones con una significación de 0,05 nos encontramos que el valor de la correlación es bajo exceptuando la correlación entre "mejorar mucho" (motivación 2) y "para saber jugar mejor" (objetivo 3) que tenía un valor de 0,313 y "entrenar con niños mejores" (motivación 3) con "porque me gusta entrenar y competir" (objetivo 6) que tenía un valor de 0,324.

Las correlaciones que también han resultado significativas pero de un modo más bajo han sido "entrenar con niños mejores" (motivación 3) con "me resulta gratificante" (objetivo 2) con un valor de 0.263 , y con "para llegar a ser tenista" (objetivo 4). 


\section{MOTIVACIÓN EN EL TENIS: UN ESTUDIO CON JÓVENES TENISTAS}

También resultaron significativas pero con un valor bajo las correlaciones entre "competir" (motivación 4) con "me resulta gratificante" (objetivo 2) con un valor de 0,263 y con "para saber jugar mejor" (objetivo 3) con un valor de 0,279.

Los resultados de la Tabla 4 son las correlaciones realizadas entre los objetivos comentados antes y las metas que eran las siguientes:

Meta 1: "ser mejor tenista"

Meta 2: "practicar bien un deporte y disfrutar con él"

Meta 3: "reconocimiento social, que mis amigos me valoren"

Meta 4: "ganar torneos importantes"

En la Tabla 4 nos encontramos como en las correlaciones anteriores con que hay correlaciones significativas al nivel 0,01 con un valor medio como en el caso de "para llegar a ser tenista" (objetivo 4) con "ganar torneos importantes" (meta 4) con un valor medio de 0,558. También el objetivo de "para llegar a ser tenista" (objetivo 4) correlaciona con "ser mejor tenista" (meta 1) teniendo un valor de 0,493 y con "practicar bien un deporte y disfrutar con él" (meta 2) siendo dicha correlación inversa con un valor de 0,332. Podemos observar que "me resulta gratificante" (objetivo 2) también correlaciona a un nivel de 0,01 con "reconocimiento social, que mis amigos me valoren" cuyo valor de la correlación es de 0,360.

Las correlaciones de significación nivel 0,05 han sido varias: "para saber jugar mejor" (objetivo 3) con "practicar bien un deporte y disfrutar con ép" (meta 2) con un valor de la correlación de 0,316. Otra correlación a este nivel de significación ha sido la que se ha producido entre "porque me gusta entrenar y competir" (objetivo 6) con "practicar bien un deporte y disfrutar con ép" con un valor de la correlación de 0,297.

Tabla 2.

Correlación Pearson entre objetivos y metas

\begin{tabular}{llrrrr}
\hline & & metas 1 & meta 2 & meta 3 & meta 4 \\
\hline objetivos 1 & Correlación de Pearson &, 153 &, 157 &, 134 &, 051 \\
& Sig. (bilateral) &, 243 &, 231 &, 309 &, 700 \\
& N & 60 & 60 & 60 & 60 \\
objetivos 2 & Correlación de Pearson &, 155 &, 095 &, $360\left(^{* *}\right)$ &, 109 \\
& Sig. (bilateral) &, 238 &, 470 &, 005 &, 409 \\
& N & 60 & 60 & 60 & 60 \\
objetivos 3 3 & Correlación de Pearson &, 128 &, $316\left(^{*}\right)$ &, 045 &, 033 \\
& Sig. (bilateral) &, 331 &, 014 &, 734 &, 805 \\
& N & 60 & 60 & 60 & 60 \\
objetivos 4 & Correlación de Pearson &, $493(* *)$ &,$- 332(* *)$ &, 145 &, $558\left(^{* *}\right)$ \\
& Sig. (bilateral) &, 000 &, 010 &, 267 &, 000 \\
& N & 60 & 60 & 60 & 60 \\
objetivos 5 & Correlación de Pearson &, 216 &, 029 &, 171 &, 165 \\
& Sig. (bilateral) &, 098 &, 826 &, 191 &, 209 \\
& N & 60 & 60 & 60 & 60 \\
objetivos 6 & Correlación de Pearson &, 044 &, $297(*)$ &, 045 &,- 014 \\
& Sig. (bilateral) &, 739 &, 021 &, 731 &, 915 \\
& N & 60 & 60 & 60 & 60 \\
\hline
\end{tabular}

** La correlación es significativa al nivel 0,01 (bilateral).

* La correlación es significante al nivel 0,05 (bilateral). 
En la Tabla 5 aparecen las correlaciones entre metas y motivación y los de las correlaciones que han resultado significativos son los que vamos a comentar a continuación.

Ha resultado significativa a un nivel 0,01 de significación la correlación entre "ser mejor tenista" (meta 1) y "entrenar con niños mejores" (motivación 3) con un valor de 0,384.

También han resultado significativas aunque a un nivel de 0,05 las correlaciones entre "practicar bien un deporte y disfrutar con ép' (meta 2) y "ser el mejor" (motivación 5) con un valor medio de $-0,312$ y "ganar torneos importantes" (meta 4) con "competir" (motivación 4) con un valor de 0,257 .

Tabla 3.

Correlación Pearson entre metas y motivación

\begin{tabular}{rlrrrrr}
\hline & & motivacion 1 & motivacion 2 & motivacion 3 & motivacion 4 & motivacion 5 \\
\hline meta 1 & Correlación de Pearson &,- 076 &,- 133 &, $384\left(^{* *}\right)$ &, 180 &, 155 \\
& Sig. (bilateral) &, 566 &, 312 &, 002 &, 169 &, 238 \\
& $\mathrm{~N}$ & 60 & 60 & 60 & 60 & 60 \\
meta 2 & Correlación de Pearson &,- 021 &, 153 &, 072 &, 196 &,$- 312\left(^{*}\right)$ \\
& Sig. (bilateral) &, 874 &, 244 &, 585 &, 133 &, 015 \\
& $\mathrm{~N}$ & 60 & 60 & 60 & 60 & 60 \\
meta 3 &, 101 &, 184 &, 251 &, 024 &,- 131 \\
& Correlación de Pearson &, 445 &, 160 &, 053 &, 854 &, 319 \\
& Sig. (bilateral) & 60 & 60 & 60 & 60 & 60 \\
meta 4 & $\mathrm{~N}$ &, 146 &, 093 &, 151 &, $257\left(^{*}\right)$ &, 210 \\
& Correlación de Pearson &, 266 &, 481 &, 249 &, 048 &, 107 \\
& Sig. (bilateral) & 60 & 60 & 60 & 60 & 60 \\
\hline
\end{tabular}

** La correlación es significativa al nivel 0,01 (bilateral).

* La correlación es significante al nivel 0,05 (bilateral).

\section{DISCUSIÓN}

En este trabajo se propuso explorar las motivaciones y necesidades de los tenistas y como afectaban a su juego y desarrollo en este deporte.

Los factores psicológicos son a día de hoy los que hacen que un tenista que está en su carrera deportiva abandone 0 continúe luchando día a día. En el tenis, de todas aquellas personas que lo practican un grupo muy reducido de ellos es el que llega a jugar a un nivel profesional.

Aquellos que tenían como objetivo llegar a ser tenista su motivación era la de ser el mejor, pudiéndose explicar porque aquellos que su meta es competir a nivel profesional su motivación no puede ser otra que ser mejor que los demás y por tanto sus expectativas de éxito en los partidos serán muy estrictas, de tal modo que una derrota para estos jugadores será crucial en futuros encuentros (Fernandez et al.,2005)

El entrenamiento es un momento en el que los jugadores tienen la oportunidad de compararse con otros jugadores y este momento también puede ser un momento estresante ya que los rivales pueden ser mejores y hacer que la autoestima que tenían de ellos mismos se vea afectada. Además a aquellos niños que les gustaba entrenar con niños mejores también estaba correlacionado con que les gustaba competir, dejando presente que la competitividad en el tenis es algo que no se puede obviar y que es durante la competición donde por fuerza los niños aprenden a saber jugar mejor (Romero, 2005)

También nos encontramos con que aquellas personas que quieren llegar a ser tenistas profesionales tienen como meta ganar torneos importantes, nada más lejos de la realidad y que el reco- 


\section{MOTIVACIÓN EN EL TENIS: UN ESTUDIO CON JÓVENES TENISTAS}

nocimiento social por parte de los amigos está relacionado con que el tenis les parezca gratificante y hace que factores como el acompañamiento en los partidos o la entrada del entrenador a pista sean necesidades que los tenistas les gustaría cambiar (Cervello et al.,2002)

Este estudio presenta diversas limitaciones que sería necesario solventar para que las repercusiones de los resultados fueran mayores. Una de ellas ha sido el tamaño muestral (61 sujetos) que se intentará aumentar en próximos estudios para poder generalizar los resultados. Otra de ellas ha sido la utilización de un cuestionario creado por nosotros y no validado, que en futuras investigaciones se intentaría validar.

En futuras investigaciones como hemos comentado, además de aumentar la muestra, podría realizarse una comparativa entre clubes, y la validación del cuestionario. Creemos que este estudio podría tener grandes aplicaciones en el campo del tenis y en una propuesta de intervención/entrenamiento en habilidades psicológicas ya que este deporte es muy individualista y los factores psicológicos podrían ayudar a proteger a los tenistas tanto de lesiones como de un bajo rendimiento.

\section{REFERENCIAS}

Balaguer, I., Castillo, I., Duda, J.L. Y García-Merita, M. (2011). Asociaciones entre la percepción del clima motivacional creado por el entrenador, orientaciones disposicionales de meta, regulaciones motivacionales y vitalidad subjetiva en jóvenes jugadores de tenis. Revista de Psicología del Deporte, 20, 131-148.

Cervelló, E., Santos-Rosa, F. J., Jiménez, R., Nerea, A. y García, T. (2002). Motivacion y ansiedad en jugadores de tenis. European Journal of Human Movement, 9, 141-161.

Fernández, J., Fernández, B., Méndez, A. y Terrados, N. (2005). La intensidad de trabajo en tenis: el entrenamiento frente a la competición. Arch. Med. Deporte, 187-192.

Gill, D. L., Gross, J. B. y Huddleton, S. (1983). Participation motivation in youth sports. International Journal of Sports Psychology, 1, 1-14.

González, G., Tabernero, B., y Márquez, S. (2000). Análisis de los motivos para participar en fútbol y en tenis en la iniciación deportiva. European Journal of Human Movement, 6, 47-66.

Moreno, J. A. y Martínez, A. (2006). Importancia de la teoría de la autodeterminación en la práctica físico-deportiva: fundamentos e implicaciones prácticas. Cuadernos de psicología del deporte, 6.

Nicholls, J. G. (1989). The competitive ethos and democratic education. Cambridge, MAAS: Hardvard University Press.

Romero, D. N. (2005). La importancia de la fortaleza mental en los deportes. Lecturas: Educación física y deportes, 90, 14.

Villamarín, F., Maurí, C., y Sanz, A. (2007). Competencia percibida y motivación durante la iniciación en la práctica de tenis. Revista de psicología del deporte, 7. 